\title{
Lockdown, Social Media exposure regarding COVID-19 and the relation with self-assessment depression and anxiety. Is the medical staff different?
}

\author{
Ariana-Anamaria Cordos ${ }^{1}$ and Sorana Daniela Bolboacă ${ }^{1}$ \\ ${ }^{1}$ Iuliu Hagieganu University of Medicine and Pharmacy Faculty of Medicine
}

July 7,2020

\begin{abstract}
Introduction. Locking the humanity in their homes, COVID-19 forced people to use the technology at hand to keep informed about the outbreak and to keep close to their loved ones. During this time, even if physical health is theoretically unaffected, keeping calm and sane can be challenging. The aim of this research was to evaluate whether exposure to COVID-19 information available in the digital space has a different impact on the mental condition of Romanian medical staff, compared to general population, particularly searching for depression and anxiety symptoms. Materials and methods. An online survey was conducted from April 6 to 16, 2020 within the Romanian users of Social Media platforms. The questionnaire assessed depression with the WHO-Five Well-Being Index, anxiety with the Generalized Anxiety Disorder Scale and Social Media exposure by asking how often the respondents saw COVID-19 related information on the most popular Social Media channels in Romania. Information about: gender, age, educational level, occupation, area of living and risk category was also collected. The risk categories were defined as no risk, medium risk, and medical staff. Results. Almost $90 \%$ of the 402 participants received daily through at least one Social Media channel information related to the COVID-19 outbreak. The Social Media Exposure significantly associated with the risk group only for Facebook and LinkedIn. However, exposure to information regarding COVID-19 was neither associated with anxiety nor depression. No significant association was identified neither between age class and self-assessed anxiety nor self-assessed depression. The self-assessment of depression was significantly more frequent as compared to self-assessment of anxiety. Conclusion. The results of this research are opposite to most of the already published literature. Depression and anxiety could not be correlated with the context of lockdown and excessive COVID-19-related information.
\end{abstract}

\section{Hosted file}

2020-07-07_COVID19-Articol_SM.docx available at https://authorea.com/users/340529/articles/ 467749-lockdown-social-media-exposure-regarding-covid-19-and-the-relation-with-selfassessment-depression-and-anxiety-is-the-medical-staff-different 


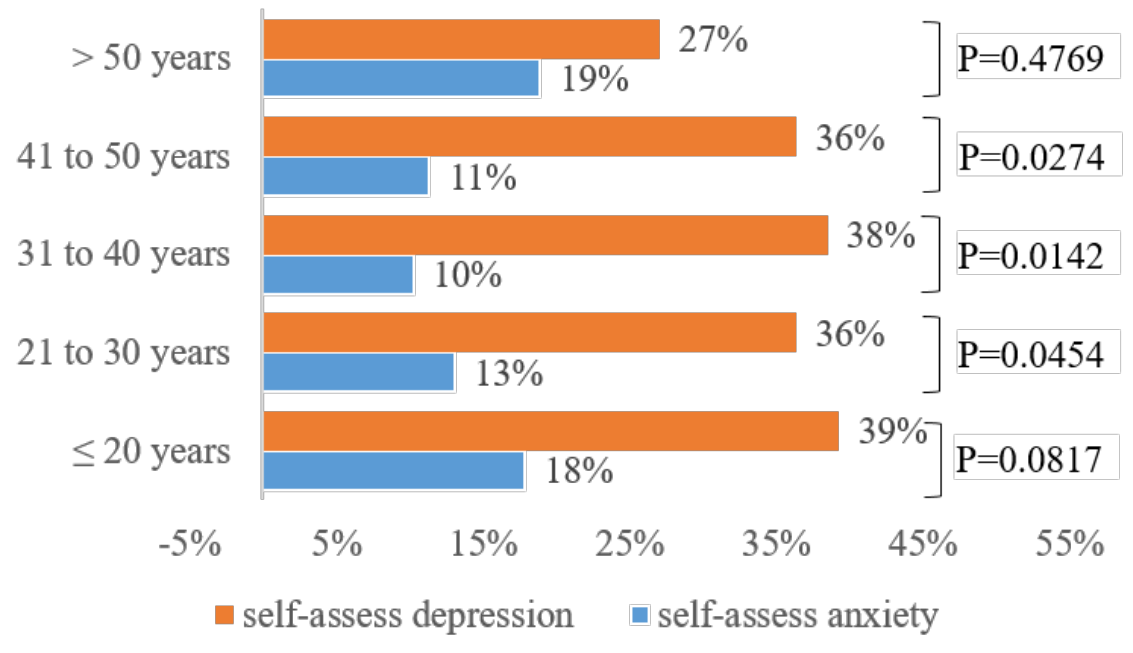

